\title{
Resistencia a antibióticos betalactámicos y quinolonas en Escherichia coli aislada de pollos broiler
}

\section{Resistance to beta-lactam antibiotics and quinolones in Escherichia coli isolated from broilers}

\author{
Edna Carvajal B. ${ }^{1}$, Egberto Rueda G. ${ }^{2}$, Martín Talavera R. ${ }^{3}$, María Torres C. ${ }^{4}$, \\ Diana López V.4, María C. Vásquez R. ${ }^{1,5}$
}

\section{Resumen}

El objetivo del estudio fue determinar la resistencia antimicrobiana de 176 cepas de Escherichia. coli aisladas de órganos de pollos broiler. Las cepas fueron desafiadas con antibióticos betalactámicos, quinolonas y fluoroquinolonas, observándose resistencia a antibióticos betalactámicos (97.7\%) y a quinolonas (86.7\%). El 71.6\% de los aislados también expresaron fenotípicamente la producción de betalactamasas de espectro extendido (ESLB). Mediante PCR se determinaron genes de resistencia para betalactámicos blaTEM, blaSHV, blaCTX-M1 y Amp-C y genes de resistencia para quinolonas qnrA, $q n r B, q n r S$. Se encontraron los genes Amp-C (74\%), blaCTX-M (65\%), blaSHV $(65 \%)$, blaTEM $(50 \%)$, qnrB $(86.4 \%)$ y qnrS $(11.9 \%)$. No se evidenció el gen $q n r A$ en las muestras analizadas. Los resultados obtenidos revelaron un gran porcentaje de resistencia a los antibióticos estudiados y la presencia de genes de resistencia en aislados de aves para consumo humano, lo cual constituye un riesgo para la salud pública.

Palabras clave: resistencia antimicrobiana, betalactamasas, quinolonas, pollo de carne

${ }^{1}$ Universidad de Santander, Facultad de Ciencias de la Salud, Grupo de investigación CliniUdes, Bucaramanga, Colombia

${ }^{2}$ Universidad de Santander, Facultad de Ciencias Exactas, Naturales y Agrícolas, Grupo de investigación en Ciencias Agropecuarias GICA, Bucaramanga, Colombia

${ }^{3}$ Universidad Autónoma del Estado de México, UAEM, Toluca Estado de México, México

${ }^{4}$ Universidad de Boyacá, Tunja, Boyacá, Colombia

${ }^{5}$ E-mail:m.vasquez@udes.edu.co

Recibido: 16 de junio de 2020

Aceptado para publicación: 22 de diciembre de 2020

Publicado: 24 de abril de 2021 
The aim of this study was to determine the antimicrobial resistance of 176 Escherichia coli strains isolated from broiler chicken organs. The strains were challenged with beta-lactam, quinolones and fluoroquinolones, observing resistance to beta-lactam antibiotics $(97.7 \%)$ and quinolones $(86.7 \%)$. The results showed that $71.6 \%$ of the isolates phenotypically expressed the production of extended spectrum beta-lactamases (ESLB). By PCR, resistance genes for beta-lactams blaTEM, blaSHV, blaCTX-MI and Amp-C and resistance genes for quinolones $q n r A, q n r B, q n r S$ were determined. The genes $A m p$ $C(74 \%)$, blaCTX-M (65\%), blaSHV (65\%), blaTEM (50\%), qnrB (86.4\%) and qnrS(11.9\%) were found. The $q n r A$ gene was not evident in the samples analysed. The results obtained revealed a large percentage of resistance to the studied antibiotics and the presence of resistance genes in isolates from poultry for human consumption, which constitutes a risk for Public Health.

Keywords: antimicrobial resistance, beta lactamases, quinolones, broiler chicken

\section{INTRODUCCIÓN}

La industria avícola en Colombia ha tenido un gran auge en los últimos años gracias a la innovación tecnológica en manejo y mejoramiento genético de las aves, aumentando la contención de patógenos mediante el uso de antibióticos como una alternativa para la prevención y tratamiento de infecciones y mediante el uso de promotores de crecimiento en las dietas (Stockwell y Duffy, 2012), logrando mayores índices de productividad (Bueno et al., 2016). Sin embargo, diversos estudios en aves han identificado la circulación de bacterias resistentes a diferentes clases de antibióticos (Mellata et al., 2018), incluyendo a bacterias con posible potencial zoonótico (Mitchel et al., 2015; Stromberg et al., 2017). Asimismo, se han encontrado determinantes genéticos implicados en la resistencia a los antimicrobianos en Escherichia coli patogénica aviar (APEC) aislados de granjas de pollos de engorde (Awad et al., 2016; Staji et al., 2018; Subedi, 2018).
Se conocen diferentes mecanismos de resistencia que pueden ser adoptados por $E$. coli, tales como modificación del sitio blanco, cambios de permeabilidad (Fábrega et al., 2008), producción de enzimas modificadoras de aminoglucósidos e incremento en la expresión de las bombas de expulsión (Tafur et $a l ., 2008)$. Las $\beta$-lactamasas de espectro extendido (ESBLs) son un grupo de enzimas transportadas en plásmidos que hidrolizan penicilinas, cefalosporinas y monobactámicos. Entre ellas están las $\beta$-lactamasas sulfhidrilo variable (SHV), temoneira (TEM), cefotaximasa (CTX-M) (Sadat et al., 2016) y $\beta$-lactamasas AmpC plasmídicas (pAmpC) (Huijbers et al., 2015; Madec et al., 2017). Las enzimas que confieren resistencia a fluoroquinolonas son también mediadas por plásmidos e incluyen las proteínas Qnr, la enzima aac(6')-Ib-cr y la bomba de eflujo QepA(Vetting et al., 2011). Se ha evidenciado asociación de la resistencia a las quinolonas con la producción de $\beta$-lactamasas de espectro extendido (ESBL) o de AmpC âlactamasas mediadas por plásmidos 
(Mendonça et al., 2016; Salgado et al., 2016) y de la incidencia de los integrones como responsables del paso de determinantes de resistencia entre microorganismos Gram negativos (Berglund, 2015)

Por otra parte, E. coli se comporta como un patógeno oportunista en situaciones de estrés y de deficiente manejo (LeStrange, 2017; Perello, 2009), donde los patotipos APEC, responsables de infección primaria en aves de corral, causan grandes pérdidas económicas a la industria avícola (Dziva y Stevens, 2008; Guabiraba y Schouler, 2015). En el humano, E. coli patógena extraintestinal (ExPEC) representa un patógeno emergente, con cepas implicadas en casos de infecciones del tracto urinario (ITU), bacteriemia y meningitis (Manges, 2016). Varios estudios han revelado características superpuestas entre APEC y ExPEC humano (Maluta, 2014; Xiangkai, 2014), lo que lleva a la hipótesis de un potencial zoonótico de las cepas de aves de corral (Cunha et al., 2017). Así mismo, la Organización Mundial de Sanidad Animal (OIE) estima que cerca del $60 \%$ de los patógenos humanos y del $75 \%$ de las enfermedades de reciente aparición son de curso zoonótico (Labro y Bryskier, 2014).

El uso excesivo e indebido de los agentes antimicrobianos en aves para consumo humano, además de las hipótesis de los linajes o raíces evolutivas comunes entre $E$. coli aviar y humana (Manges, 2016), contribuyen al desarrollo y la propagación de la resistencia a antibióticos (Hussain et al., 2017), lo que lleva a la aparición de patógenos multirresistentes, los cuales pueden presentar la transmisión cruzada al humano (MoulinSchouleur et al., 2016), convirtiéndose en un grave e importante problema de salud pública. Esta situación obliga a mejorar las prácticas al margen de las recomendaciones de producción, control de calidad e inocuidad alimentaria (FAO, 2011). El presente estudio tuvo como objetivo determinar la resistencia a antibióticos $\beta$-lactámicos y quinolonas y establecer la presencia de genes involucrados en aislamientos de E. coli obtenidos de pollos broiler provenientes de granjas de producción avícola de Santander, Colombia.

\section{Materiales y Métodos}

Se realizó un estudio descriptivo de corte transversal, recolectando 400 muestras de varios órganos (tráquea, intestinos, sacos aéreos abdominales y claviculares, pericardio, bolsa de Fabricio y páncreas) en necropsias de 200 pollos de engorde sanos de cinco edades (12, 18, 21, 36 y 42 días). Todos los especímenes provinieron de granjas avícolas del departamento de Santander, Colombia.

Las muestras fueron inoculadas en caldo Infusión Cerebro Corazón (BHI) para posterior siembra en agar MacConkey. Las colonias fermentadoras de lactosa en agar MacConkey se identificaron bioquímicamente (Borie et al., 1997), con el kit BBL ${ }^{\mathrm{TM}}$ Crystal $^{\mathrm{TM}} \mathrm{E} / \mathrm{NF}$ y se corroboraron mediante PCR para detectar el gen uidA que codifica la enzima 1- glucuronidasa (Molina et al., 2015), la cual es específica para E. coli, utilizando cebadores propuestos por Hessain $e t$ al. (2015).

\section{Susceptibilidad a Antibióticos}

A los aislados identificados como E. coli se les realizó la prueba de Kirby Bauer (CLSI, 2017) con antibióticos pertenecientes a grupos de las quinolonas y $\beta$-lactámicos. Cada cepa se expuso frente a sensidiscos impregnados con los siguientes antibióticos: ampicilina $10 \mu \mathrm{m}$, amoxacilina/ácido clavulánico 20/10 $\mu \mathrm{m}$, piperacilina $100 \mu \mathrm{m}$, piperacilina/ tazobactam $100 / 10 \mu \mathrm{m}$, cefalotina $30 \mu \mathrm{m}$, cefuroxime $30 \mu \mathrm{m}$, cefoxitin $30 \mu \mathrm{m}$, cefotaxime $30 \mu \mathrm{m}$, ceftazidime $30 \mu \mathrm{m}$, ceftriaxona $30 \mu \mathrm{m}$, cefepime $30 \mu \mathrm{m}$, ácido nalidíxico $30 \mu \mathrm{m}$, norfloxacina $10 \mu \mathrm{m}$ y ciprofloxacino $5 \mu \mathrm{m}$. Luego se evaluó la susceptibilidad de las cepas siguiendo las recomendaciones del CLSI (2017). 
Cuadro 1. Condiciones de PCR para amplificación de los genes de resistencia en cepas de Escherichia coli fenotipo ESLB

\begin{tabular}{|c|c|c|c|c|c|c|c|}
\hline $\begin{array}{l}\bar{D} \\
\stackrel{0}{0}\end{array}$ & 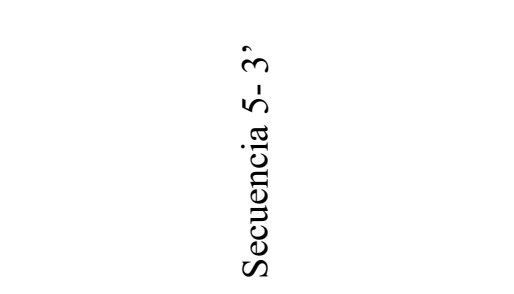 & 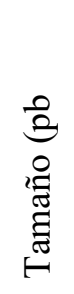 & 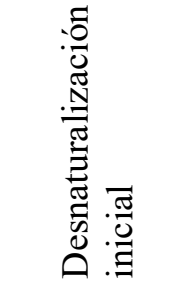 & $\frac{\stackrel{0}{0}}{\stackrel{0}{0}}$ & 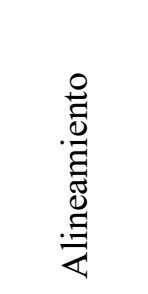 & 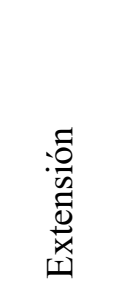 & 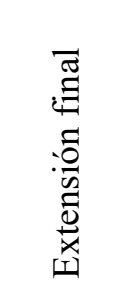 \\
\hline 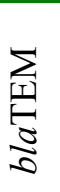 & $\begin{array}{l}\text { 5'-AAACGCTGGTGAAA } \\
\text { GTA 3' } \\
\text { 5'-AGCGATCTGTCTAT 3' }\end{array}$ & 239 & $94^{\circ} \mathrm{C}, 30 \mathrm{~s}$ & 35 & $\begin{array}{c}49^{\circ} \mathrm{C}, 1 \\
\min \end{array}$ & $\begin{array}{l}72^{\circ} \mathrm{C}, \\
1 \mathrm{~min}\end{array}$ & $\begin{array}{l}72^{\circ} \mathrm{C}, \\
10 \mathrm{~min}\end{array}$ \\
\hline 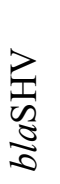 & $\begin{array}{l}\text { 5'ATGCGTTATATTCGCCTG } \\
\text { TG 3' } \\
\text { 5'-TGCTTTGTTATTCGGG } \\
\text { CCAA 3' }\end{array}$ & 241 & $94^{\circ} \mathrm{C}, 30 \mathrm{~s}$ & 35 & $56^{\circ} \mathrm{C}$ & $\begin{array}{l}72{ }^{\circ} \mathrm{C} \\
1 \mathrm{~min}\end{array}$ & $\begin{array}{l}72^{\circ} \mathrm{C} \text {, } \\
10 \mathrm{~min}\end{array}$ \\
\hline 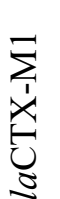 & $\begin{array}{l}\text { 5'-GACGATGTCACTGGC } \\
\text { TGAGC 3' } \\
\text { 5'-AGCCGCCGACGCTA } \\
\text { ATACA 3' }\end{array}$ & 499 & $94^{\circ} \mathrm{C}, 30 \mathrm{~s}$ & 35 & $58^{\circ} \mathrm{C}$ & $\begin{array}{l}72{ }^{\circ} \mathrm{C}, \\
1 \mathrm{~min}\end{array}$ & $\begin{array}{l}72^{\circ} \mathrm{C}, \\
10 \mathrm{~min}\end{array}$ \\
\hline $\begin{array}{l}\text { Ù } \\
\text { 家 }\end{array}$ & $\begin{array}{l}\text { 5'-ATCAAAACTGGCAG } \\
\text { CCG-3' } \\
\text { 5'-GAGCCCGTTTTATGC } \\
\text { ACCCA-3' }\end{array}$ & 170 & $94^{\circ} \mathrm{C}, 30 \mathrm{~s}$ & 35 & $56.9^{\circ} \mathrm{C}$ & $\begin{array}{l}72^{\circ} \mathrm{C}, \\
1 \mathrm{~min}\end{array}$ & $\begin{array}{l}72^{\circ} \mathrm{C}, \\
10 \mathrm{~min}\end{array}$ \\
\hline
\end{tabular}

Fuente: Protocolo de estandarización de Paterson et al. (2003), modificado por Velandia et al. (2016)

Genes de Resistencia blaTEM, blaSHV, blaCTX-M1, Amp-C

Mediante PCR convencional se realizó la amplificación a las cepas de E. coli. Se utilizó la cepa Klebsiella pneumoniae ATCC 700603 control positivo y la cepa $E$. coli ATCC 25922 como control negativo. La Figura 1 presenta las condiciones del PCR.
La caracterización de los genes qnrA, $q n r B$ y $q n r S$ en cada una de las cepas de $E$. coli se realizó de acuerdo con el protocolo estandarizado por Aguilar et al. (2015). Los resultados de PCR se visualizaron en gel de agarosa al $1 \%$ con tinción de Safeview classic. Se utilizó un transiluminador UltraSlim Led Illuminator (ABM). La presencia de los genes en evaluación se determinó en las cepas resultantes. 


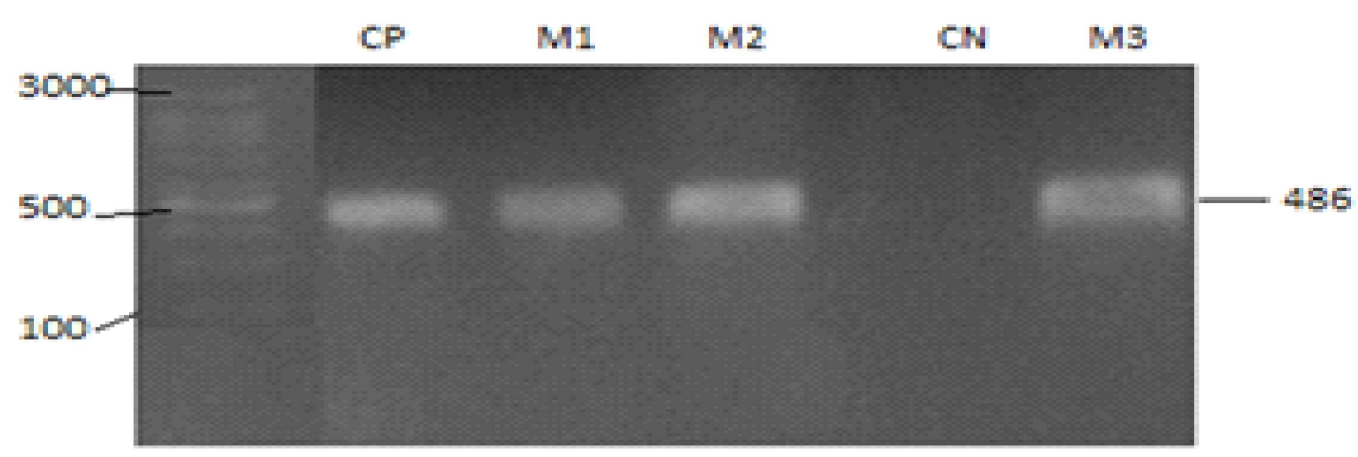

Figura 1. Detección del gen uidA.Gel de agarosa (1\%). Carril 1, 100 bp DNA ladder New England Bio Labs. Carril 2, control positivo. Carril 3, Muestra 1 uidA +. Carril 4. Muestra 2 uidA +. Carril 5, Control negativo. Carril 6, Muestra 3 uidA+

\section{Bienestar Animal}

Se siguieron todas las pautas internacionales, nacionales e institucionales para el cuidado y uso de los animales. Se tuvieron en cuenta todas las normas de bienestar animal de acuerdo con la Ley 1774 del 6 de enero de 2016, Art. 339B, Párrafo 1 de la Republica de Colombia (2016). El estudio contó, además, con el aval del comité de ética de la Unversidad de Boyacá. El manuscrito no contiene estudios clínicos ni datos de pacientes.

\section{Resultados}

Se obtuvieron 176 aislados de E. coli de las 400 muestras colectadas. La resistencia de los aislados a antibióticos betalactámicos fue del $97.7 \%$ y a quinolonas del $86.7 \%$. Los antibióticos del grupo de betalactámicos probados y el porcentaje de resistencia para cada uno de ellos fueron: ampicilina $100 \%$, cefuroxime $98.9 \%$, amoxacilina - ácido clavulánico $98.9 \%$, cefalotina $97.7 \%$, piperacilina tazobactam $97 \%$, cefoxitina $96.6 \%$, cefotaxime $96.6 \%$, piperacilina: $95.5 \%$, ceftazidime $93.2 \%$, ceftriaxona $92.0 \%$, y cefepime $83.0 \%$. Fren- te a las quinolonas se obtuvo resistencia para ácido nalidíxico en el 100\% de los aislados, a la ciprofloxacina en el $83 \%$ y a la norfloxacina en el $77.3 \%$ (Figura 2).

Los resultados de la prueba confirmatoria evidencian resultado positivo para el $71.6 \%$ de los aislamientos, los cuales expresaron fenotípicamente ESLB (Figura 3).

Los datos de PCR para genes que codifican la resistencia tipo ESLB revelaron que el gen $A m p C$ (74\%) fue el más frecuente en los aislados de E. coli, seguido de los genes blaSHV y blaCTXM (65\%) y el gen blaTEM (50\%). El 34.9\% de las cepas amplificaron los cuatro genes, $25.6 \%$ tres, $20.9 \%$ dos y $4.7 \%$ un gen, y el restante $13.9 \%$ no mostró amplificación. Por otra parte, los datos de PCR para genes que codifican resistencia a las quinolonas mostró al gen $q n r B$ con el $86.4 \%$ y al $q n r S$ con el $11.9 \%$ de los aislados de E. coli, mientras que no se evidenció la presencia del gen qnrA (Figura 4).

Se obtuvo las amplificaciones de los genes con el protocolo establecido revelando bandas de 700, 700, 500 y $550 \mathrm{pb}$ para los genes blaTEM, blaSHV, blaCTX-M1 y $A m p C$, respectivamente (Figuras 5 y 6 ). 


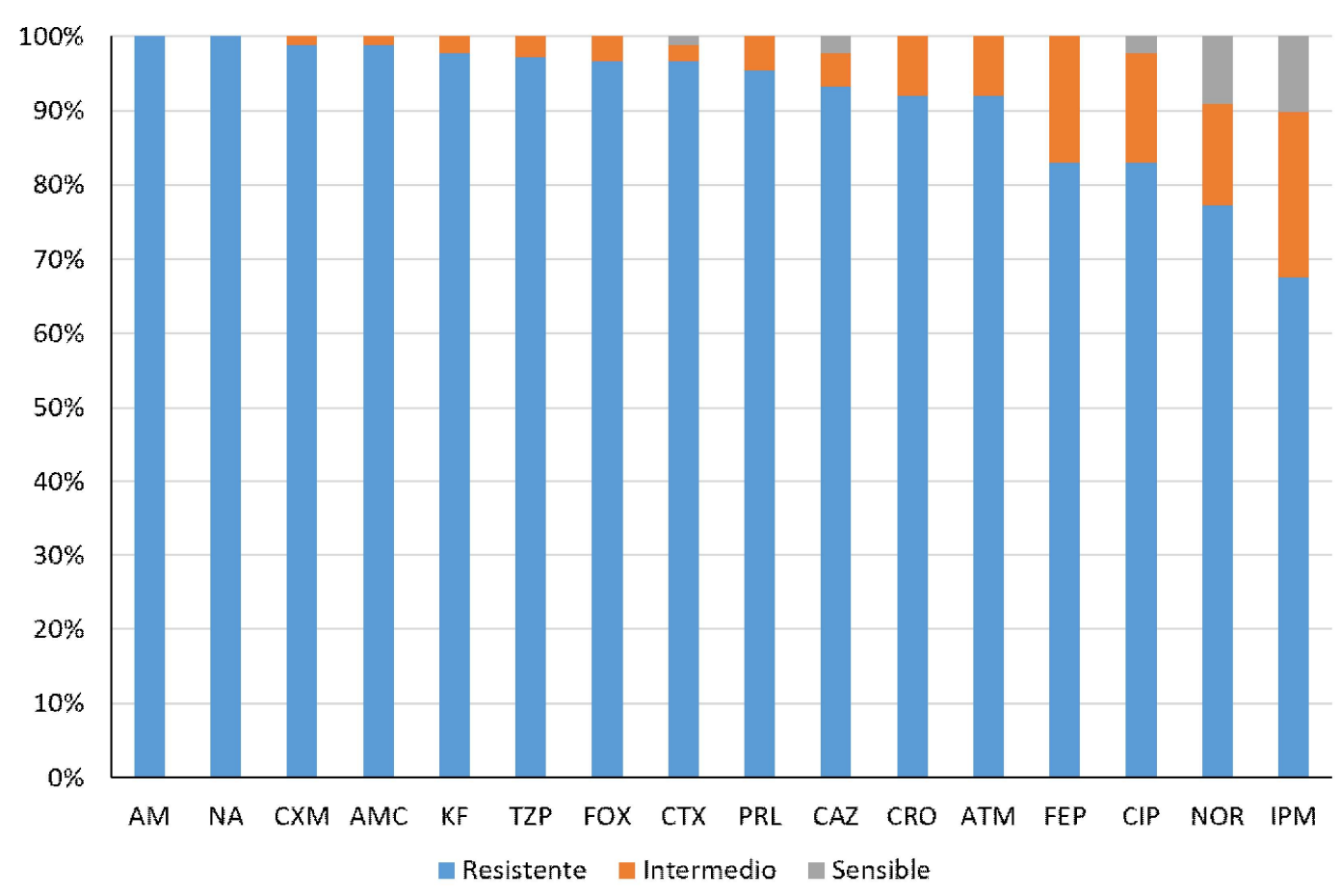

Figura 2. Patrón de resistencia de 176 aislamientos de E. coli a 16 agentes antimicrobianos: AM: ampicilina; NA: ácido nalidíxico; CXM: cefuroxima; AMC: amoxicilina/ácido clavulánico; KF: cefalotina; TZP: piperacilina/tazobactam; FOX: cefoxitina; CTX: cefotaxime; PRL: piperacilina; CAZ: ceftazidima; CRO: ceftriaxona; ATM: aztreonam; FEP: cefepime; CIP: ciprofloxacina, NOR: norfloxacina

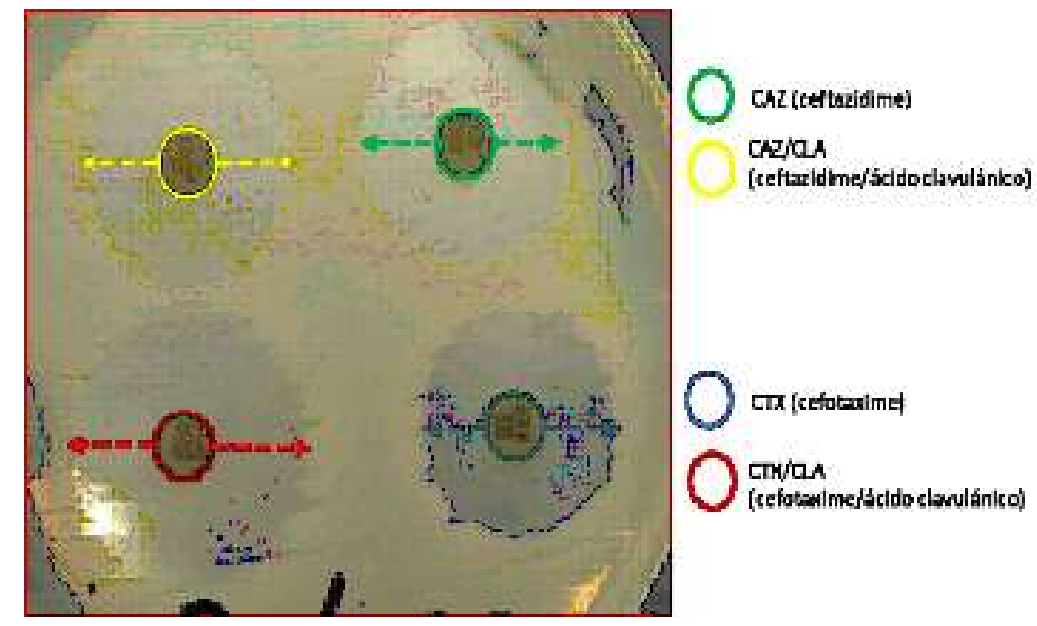

Figura 3. Prueba confirmatoria fenotipo ESLB: método del doble disco. Se observa el aumento del halo en más de $5 \mathrm{~mm}$ con los sensidiscos de mezcla de cefalosporina y ácido clavulánico, con relación al disco de cefalosporina sola (CLSI, 2017) 


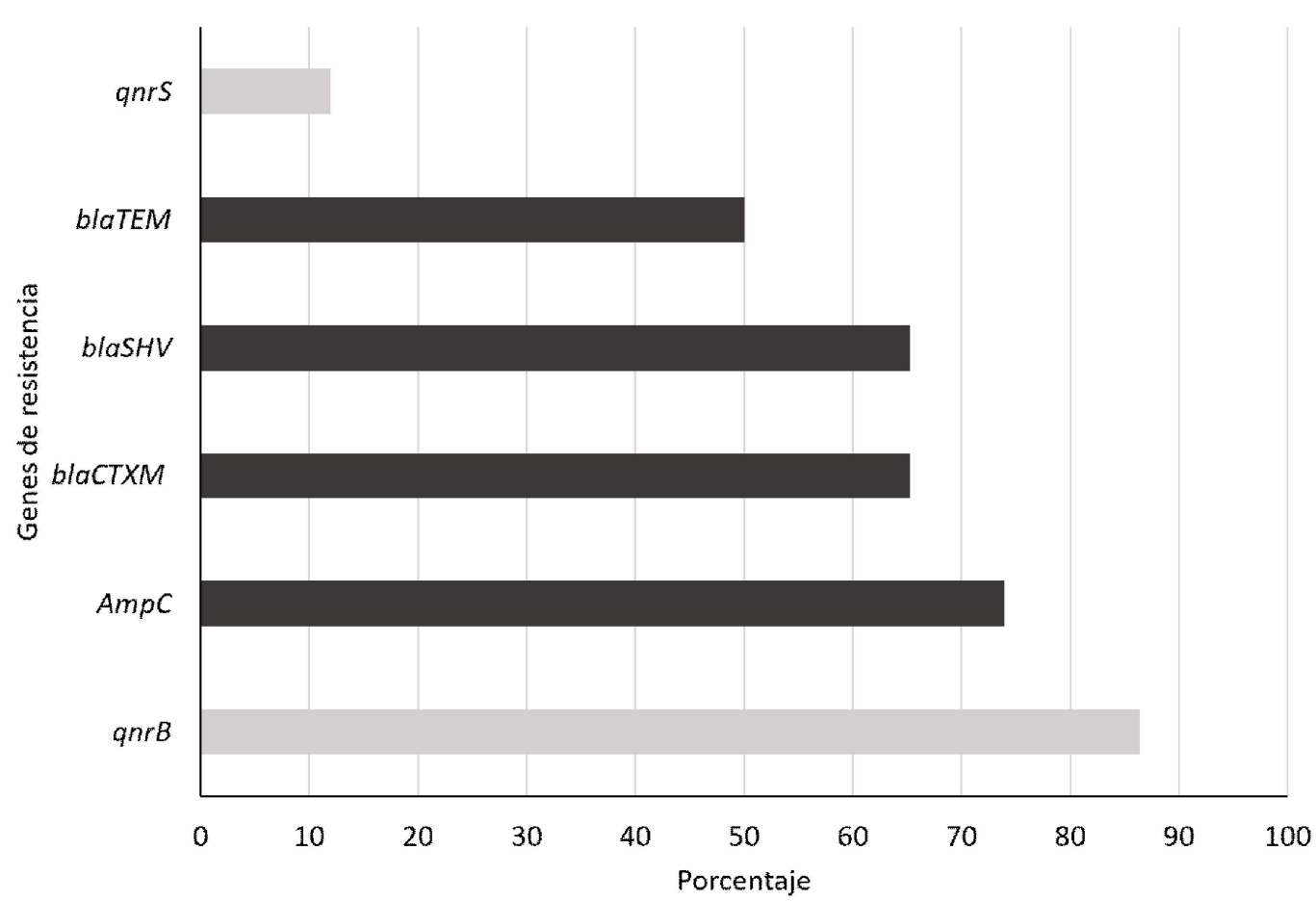

Figura 4. Porcentaje de genes de resistencia de los aislados de E. coli a antibióticos betalactámicos: genes $A m p C$; blaSHV; blaCTXM y blaTEM y quinolonas: genes $q n r S$ y $q n r B$

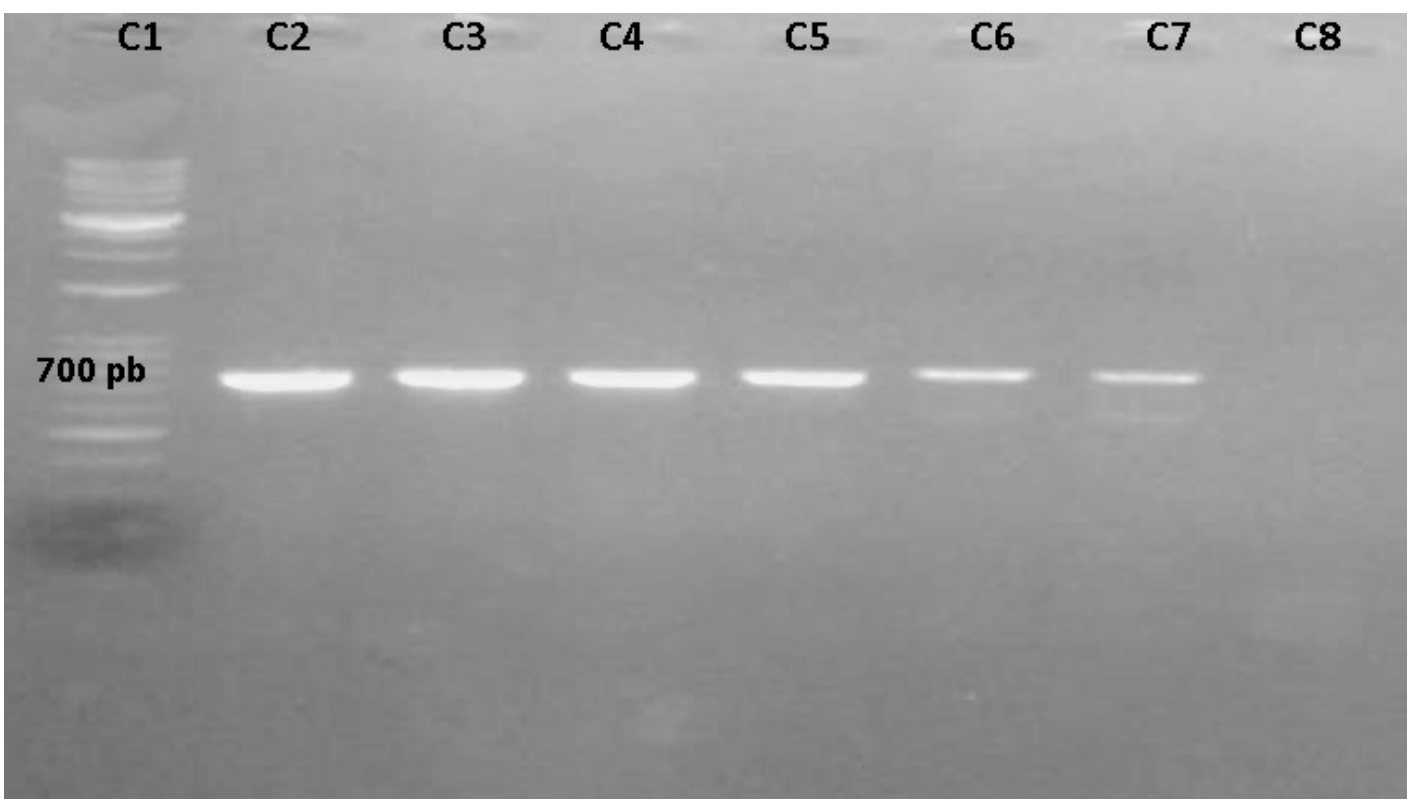

Figura 5. Gel de electroforesis del gen blaSHV: $\mathrm{C} 1: 1.5 \mathrm{~Kb}$; $\mathrm{C} 2$ : Control positivo; $\mathrm{C} 3$ : $\mathrm{Mx} 1 ; \mathrm{C} 4$ : $\mathrm{Mx} 2$; C5: Mx3; C6: Mx4; C7: Mx5; C8: control negativo 


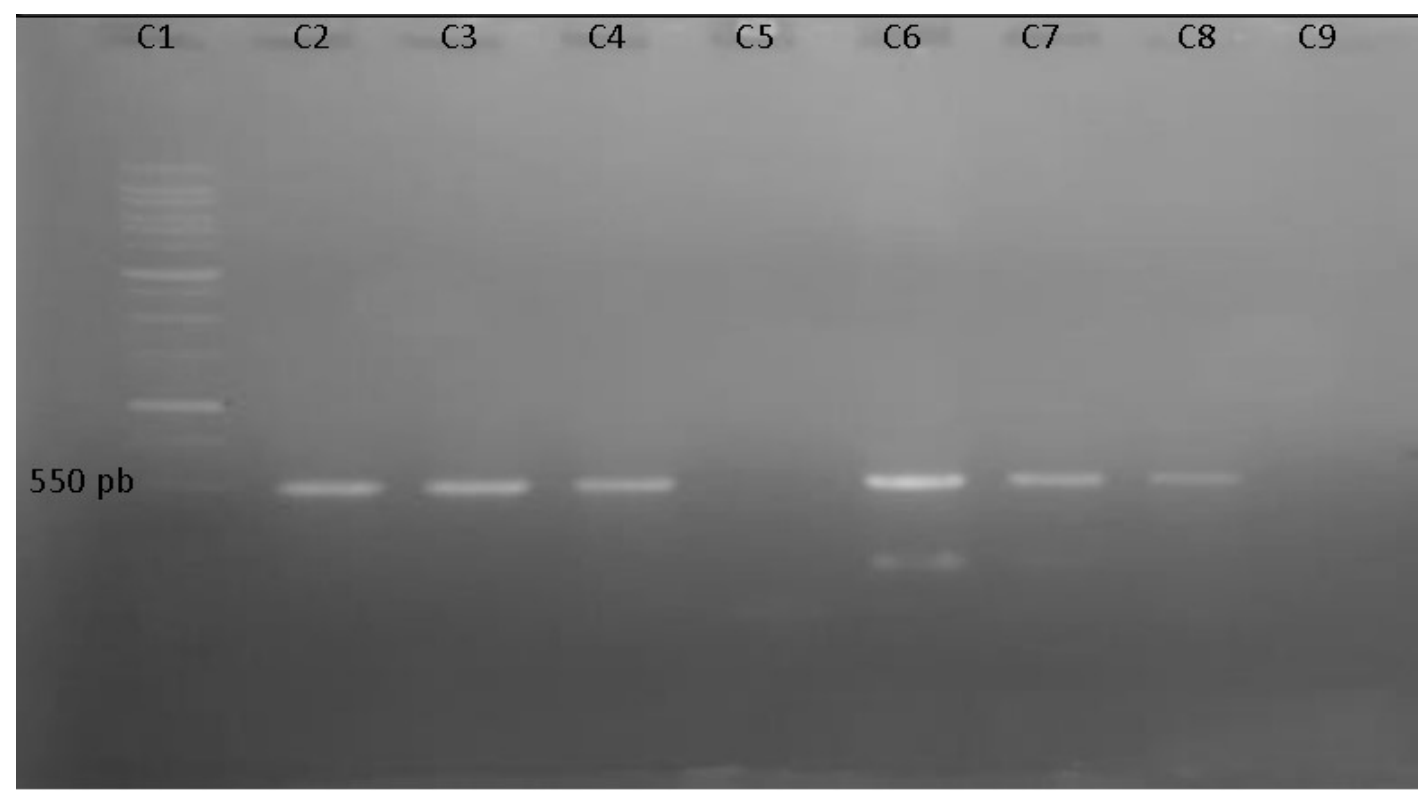

Figura 6. Gel de electroforesis de gen $A m p C$ : $\mathrm{C} 1: 1 \mathrm{~Kb}, \mathrm{C} 2$ : Control positivo; C3: Mx1; C4: Mx2; C5: Mx3; C6: Mx4; C7: Mx5; C8: Mx6; C9: Control negativo

\section{Discusión}

El estudio evidenció la multirresistencia de las cepas de E. coli a los antibióticos evaluados por el método de difusión en disco. Todas las cepas presentaron resistencia a la ampicilina y el $95 \%$ a todos los betalactámicos enfrentados, resaltando la producción de ESBL en el $71 \%$ de las cepas. En este sentido, diversos estudios muestran la presencia de bacterias portadoras de genes que codifican resistencia a antibióticos betalactámicos (Cuadro 2).

Se observó una elevada prevalencia del gen $A m p C$, que codifica para la resistencia a las cefamicinas (cefoxitina y cefotetan), oximinocefalosporinas (ceftazidima, cefotaxime y ceftriaxona), monobactámicos (aztreonam) y aminopenicilinas combinadas con inhibidores de betalactamasas (amoxicilina-ácido clavulánico, ampicilina-sulbac- tam). Este gen se ha caracterizado por estar presente en especies y géneros bacterianos como Enterobacter, Providencia, Morganella morganii, Serratia marcescens y Citrobacter freundii; sin embargo, en $E$. coli puede identificarse aunque con una expresión baja (Lopez et al., 2016). Muzo (2017) comprobó el fenotipo de cepas ESLB $(80 \%)$ y $A m p C$ (20\%), lo cual evidencia la amplia difusión de bacterias resistentes a antibióticos betalactámicos en pollos broiler de Galápagos destinados para el consumo humano. Los hallazgos de Castellanos (2017) en aves de corral en Colombia indican una $100 \%$ de prevalencia del gen bla TEM, así como resistencia causada por genes como bla CMY-2 y bla $S H V-12$. La diseminación de estas cepas resistentes está dada por plásmidos IncI1/ST12 homogéneos, los que sugiere que la propagación de la resistencia está mediada principalmente por la transferencia horizontal de genes (Castellanos et al., 2017). 
Cuadro 2. Estudios de genes de resistencia a antibióticos betalactámicos

\begin{tabular}{|c|c|c|c|c|c|}
\hline $\begin{array}{l}\text { Aislamientos } \\
\text { E. coli ESLB }\end{array}$ & $\begin{array}{l}C T X-M \\
(\%)\end{array}$ & $\begin{array}{l}\text { SHV } \\
(\%)\end{array}$ & $\begin{array}{l}\text { TEM } \\
(\%)\end{array}$ & $\begin{array}{l}A m p C \\
(\%)\end{array}$ & Referencia \\
\hline 196 & 59.1 & & & & Abreu et al. (2014) \\
\hline 134 & $\begin{array}{l}\text { 8.9 CTX-M-1 } \\
17.2 C T X-M-2 \\
7.5 C T X M-9\end{array}$ & 67.7 & 77.6 & 11.2 & $\begin{array}{l}\text { Ghodousiet al. } \\
\text { (2015) }\end{array}$ \\
\hline 78 & 89.0 & 47.0 & 27.0 & 12.0 & Reich et al. (2013) \\
\hline 176 & 65.2 & 65.2 & 50.0 & 73.9 & Este estudio \\
\hline
\end{tabular}

En la resistencia de $100 \%$ encontrada para las cepas al NA y $83 \%$ a la CIP, el gen $q n r B$ amplificó en el $86.4 \%$ de las cepas aisladas y el $q n r S$ en el $11.9 \%$, sin evidenciarse el gen $q n r A$. Todas las cepas con resistencia presentaban 1 o 2 genes, similares a los resultados de Cota-Rubio (2014) con E. coli aislada de pollos enfermos, quienes obtuvieron un porcentaje similar de resistencia al NA y de $79 \%$ a CIP. De igual forma, el $9 \%$ de los aislamientos portadores del gen $q n r$ presentaron sensibilidad intermedia a la ciprofloxacina y a la norfloxacina en las pruebas de difusión en disco.

Aguilar et al. (2015) encontraron en aislados de E. coli provenientes de canales de bovinos, resistencia al ácido nalidíxico (64\%), seguida de ampicilina (32\%), ciprofloxacino $(10 \%)$, ceftazidima y cefotaxima (ambos con $1.3 \%$ ), porcentajes de resistencia menores a los obtenidos en este trabajo. La alta circulación de bacterias resistentes en la producción avícola se manifiesta; así Bezerra et al. (2016) reporta resistencia a más de tres grupos de antibióticos, Hernández et al. (2017) encontraron $93.3 \%$ de multirresistencia y Mainali et al. (2013) identificaron $79.2 \%$ de resistencia a uno o más de los antibióticos evaluados, donde el $54.3 \%$ fueron resistentes a tres o más antimicrobianos y el $10.8 \%$ a cinco o más antimicrobianos.

\section{Conclusiones}

Los aislados de E. coli de muestras de órganos de pollos broiler de granjas avícolas de Santander, Colombia, portan genes de resistencia a antibióticos betalactámicos como Amp-C (74\%), blaCTX-M (65\%), blaSHV (65\%) y blaTEM (50\%), y a quinolonas qnrB (86.4\%) y qnrS (11.9\%), pero sin evidenciar el gen $q n r A$.

\section{Agradecimientos}

Los investigadores expresan su agradecimiento a la Universidad de Santander (UDES), a la Universidad de Boyacá y a la Universidad Autónoma del Estado de México; asimismo, a las empresas avícolas que apoyaron el proceso.

\section{Literatura Citada}

1. Aguilar-Montes de Oca S, TalaveraRojas M, Soriano-Vargas E, BarbaLeón J, Vazquez-Navarrete J. 2015. Determination of extended spectrum âlactamases/AmpC $\beta$-lactamases and plasmid-mediated quinolone resistance in Escherichia coli isolates obtained from bovine carcasses in Mexico. Trop Anim Health Pro 47: 975-981. doi: 10.1007/ s11250-015-0818-3 
2. Awad A, Arafat N, Elhadidy M. 2016. Genetic elements associated with antimicrobial resistance among avian pathogenic Escherichia coli. Ann Clin Microb Anti 15: 59. doi: 10.1186/s12941016-0174-9

3. Berglund B. 2015. Environmental dissemination of antibiotic resistance genes and correlation to anthropogenic contamination with antibiotics. Infect Ecol Epidemiol 5: 28564. doi: 10.3402/ iee.v5.28564

4. Bezerra W, da Silva I, Vanconcelos R, Machado D, Lopes E, Lima $S$, Teixeira R. 2016. Isolation and antimicrobial resistance of Escherichia coli and Salmonella enterica subsp. enterica $(\mathrm{O}: 6,8)$ in broiler chickens. Acta Sci Vet 44: 1364. doi: 10.22456/ 1679-9216.80957

5. Borie C, Monreal Z, Guerrero P, Sánchez ML, Martínez J, Arellano C, Prado V. 1997. Prevalencia y caracterización de Escherichia coli enterohemorrágica aisladas de bovinos y cerdos sanos faenados en Santiago, Chile. Arch Med Vet 29: 205-212. doi: 10.4067/ S0301-732X1997000200005

6. Bueno DJ, López N, Rodríguez F, Procura F. 2016. Producción de pollos parrilleros en países sudamericanos y planes sanitarios nacionales para el control de Salmonella en dichos animales. Agron Noroeste Argentino 36: 11-37.

7. Castellanos LR, Donado-Godoy P, León M, Clavijo V, Arevalo A, Bernal JF, Timmerman AJ, et al. 2017. High heterogeneity of Escherichia coli sequence types harbouring ESBL/AmpC genes on IncI1 plasmids in the Colombian poultry chain. Plos One 12: e0170777. doi: 10.1371/journal.pone.0170777

8. [CLSI] Clinical and Laboratory Standards Institute. 2017. Performance standards for antimicrobial susceptibility testing. [Internet]. Available in: http://file.qums.ac.ir/repository/mmrc/ clsi\%202017.pdf
9. Cota-Rubio E, Hurtado-Ayala L, Pérez E, Alcántara L. 2014. Resistencia a antibióticos de cepas bacterianas aisladas de animales destinados al consumo. Rev Iberoam Cienc 1: 75-85.

10. Cunha MP, Saidenberg AB, Moreno AM, Ferreira AJ, Vieira MA, Tardelli TA, Knobl T. 2017. Pandemic extra-intestinal pathogenic Escherichia coli (ExPEC) clonal group O6-B2-ST73 as a cause of avian colibacillosis in Brazil. Plos One 12: e0178970. doi: 10.1371/ journal.pone. 0178970

11. Dziva F, Stevens MP. 2008. Colibacillosis in poultry: unravelling the molecular basis of virulence of avian pathogenic Escherichia coli in their natural hosts. Avian Pathol 37: 355-366. doi: 10.1080/ 03079450802216652

12. Fàbrega A, Sánchez-Céspedes J, Soto $S$, Vila J. 2008. Quinolone resistance in the food chain. Int J Antimicrob Ag 31: 307-315. doi: 10.1016/j.ijantimicag.2007.12.010

13. [FAO] Organización de las Naciones Unidas para la Alimentación y la Agricultura. 2011. Boletín de enfermedades transfronterizas de los animales. [Internet]. Disponible en: http:// www.fao.org/3/i0574s/i0574s00.htm

14. Ghodousi A, Bonura C, Di Noto AM, Mammina $C$. 2015. Extendedspectrum ß-lactamase, AmpC-producing, and fluoroquinolone-resistant Escherichia coli in retail broiler chicken meat, Italy. Foodborne Pathog Dis 12: 619-625. doi: 10.1089/fpd.2015.1936

15. Guabiraba R, Schouler C. 2015. Avian colibacillosis: still many black holes. FEMS Microbiol Lett 362: fnv118. doi: 10.1093/femsle/fnv118

16. Hernández R, Báez M, Alfonso P, Espinosa, I. 2017. Susceptibilidad antimicrobiana y formación de biopelícula en aislados de Escherichia coli procedentes de gallinas ponedoras. Rev Salud Anim 39: 1-13. 
17. Hessain AM, Al-Arfaj AA, Zakri AM, El-Jakee JK, Al-Zogibi OG, Hemeg HA, Ibrahim IM. 2015. Molecular characterization of Escherichia coli O157:H7 recovered from meat and meat products relevant to human health in Riyadh, Saudi Arabia. Saudi J Biol Sci 22: 725-729. doi: 10.1016/j.sjbs.2015.06.009

18. Huijbers PM, van Hoek AH, Graat EA, Haenen AP, Florijn A, Hengeveld $P D$, van Duijkeren E. 2015. Methicillin-resistant Staphylococcus aureus and extended-spectrum and AmpC $\beta$-lactamase-producing Escherichia coli in broilers and in people living and/or working on organic broiler farms. Vet Microbiol $176: 120-125$. doi: 10.1016/j.vetmic.2014.12.010

19. Hussain A, Shaik S, Ranjan A, Nandanwar N, Tiwari SK, Majid M, Baddam R, et al. 2017. Risk of transmission of antimicrobial resistant Escherichia coli from commercial broiler and free-range retail chicken in India. Front Microbiol 8: 2120. doi: 10.3389/fmicb.2017.02120

20. Labro MT, Bryskier JM. 2014. Antibacterial resistance: an emerging 'zoonosis'? Expert Rev Anti-Infe 12: 1441-1461. doi: 10.1586/14787210.2014.976611

21. LeStrange K, Markland SM, Hoover DG, Sharma M, Kniel KE. 2017. An evaluation of the virulence and adherence properties of avian pathogenic Escherichia coli. One Health 4: 22-26.

22. Lopez D, Torres M, Prada C. 2016. Genes de resistencia en bacilos Gram negativos: impacto en la salud pública en Colombia. Universidad y Salud 18: 190-202.

23. López DP, Torres MI, Castañeda LM, Prada CF. 2016. Determinación de genes que codifican la resistencia de betalactamasas de espectro extendido en bacilos Gram negativos aislados de urocultivos. Rev Invest Salud Univ Boyacá 3: 107-126.

24. Madec JY, Haenni M, Nordmann P, Poirel L. 2017. Extended-spectrum $\beta$ lactamase/AmpC- and carbapenemaseproducing Enterobacteriaceae in animals: a threat for humans? Clin Microbiol Infec 23: 826-833. doi: 10.1016/j.cmi.2017.01.013

25. Mainali C, McFall M, King R, Irwin R. 2013. Evaluation of antimicrobial resistance profiles of Escherichia coli isolates of broiler chickens at slaughter in Alberta, Canada. J Food Protect 76: 2045-2051. doi: 10.4315/0362-028X.JFP13-203

26. Maluta RP, Logue CM, Casas MR, Meng T, Guastalli EAL, Rojas TC, Montelli AC, et al. 2014. Overlapped sequence types (STs) and serogroups of avian pathogenic (APEC) and human extra-intestinal pathogenic (ExPEC) Escherichia coli isolated in Brazil. Plos One 9: e105016. doi: 10.1371/journal.pone. $0105016 . t 002$

27. Manges AR. 2016. Escherichia coli and urinary tract infections: the role of poultry-meat. Clin Microbiol Infec 22: 122-129. doi: 10.1016/j.cmi.2015.11.010

28. Mellata M, Johnson JR, Curtiss R. 2018. Escherichia coli isolates from commercial chicken meat and eggs cause sepsis, meningitis and urinary tract infection in rodent models of human infections. Zoonoses Public Hlth 65: 103113. doi: $10.1111 /$ zph.12376

29. Mendonça N, Figueiredo $R$, Mendes C, Card RM, Anjum MF, da Silva GJ. 2016. Microarray evaluation of antimicrobial resistance and virulence of Escherichia coli isolates from Portuguese poultry. Antibiotics 5: 4. doi: 10.3390/antibiotics5010004 
30. Mitchell NM, Johnson JR, Johnston B, Curtiss R, Mellata M. 2015. Zoonotic potential of Escherichia coli isolates from retail chicken meat products and eggs. Appl Environ Microb 81: 11771187. doi: 10.1128/AEM.03524-14

31. Molina F, López-Acedo, Tabla R, Gómez A, Rebollo JE. 2015. Improved detection of Escherichia coli and coliform bacteria by multiplex PCR. BMC Biotechnol 15: 48.

32. Moulin-Schouleur M, Schouler C, Tailliez P, Kao MR, Brée A, Germon P, Oswald E, et al. 2006. Common virulence fac-tors and genetic relationships between O18:K1:H7 Escherichia coli isolates of human and avian origin. J Clin Microbiol 44: 3484-3492. doi: 10.1128/ JCM.00548-06

33. Мuzo S. 2017. Aislamiento y fenotipificación de cepas Blee y Ampc de Escherichia coli procedentes de pollos broiler en la isla Santa Cruz provincia de Galápagos. Tesis de Médico Veterinario Zootecnista. Ecuador: Univ. Central del Ecuador. $62 \mathrm{p}$.

34. Paterson DL, Hujer KM, Hujer AM, Yeiser B, Bonomo MD, Rice LB, Bonomo RA; International Klebsiella Study Group. 2009. Extended-spectrum beta-lactamases in Klebsiella pneumoniae bloodstream isolates from seven countries: dominance and widespread prevalence of SHV- and CTX-M-type beta-lactamases. Antimicrob Agents $\mathrm{Ch}$ 47: 3554-3560. doi: 10.1128/aac.47.11.3554-3560.2003

35. Perello M. 2009. Detección y caracterización de aislados de Escherichia coli de origen clínico y fecal en gallinas ponedoras. Tesis Doctoral. Madrid, España: Univ. Complutense de Madrid. $184 \mathrm{p}$.

36. Reich F, Atanassova V, Klein G. 2013. Extended-spectrum â-lactamase- and AmpC-producing enterobacteria in healthy broiler chickens, Germany. Emerg Infect Dis 19: 1253125-9. doi: 10.3201/eid1908.120879.
37. República de Colombia. 2016. Ley 1774 del 6 enero de 2016. [Internet]. Disponible en: Obtenido de http:// es.presidencia.gov.co/normativa/normativa/LEY\%201774\%20DEL\%206\%20DE\%20ENERO\%20DE\%202016.pdf

38. Sadat S, Goudarzi M, Sabzehali F. 2016. Relation between blaTEM, blaSHV and blaCTX-M genes and acute urinary tract infetions. J Acute Dis 5: 71-76.

39. Salgado-Muñoz TG, MoronesEsquivel I, Gonzaga-López, TI, Matamoros-Mejía AP, Terán-González JO, Arteaga-Vásquez S, CastroD'Franchis LJ, et al. 2016. Resistencia a quinolonas en enterobacterias con betalactamasa. Med Interna Méx 32: 277-283.

40. Staji H, Tonelli A, Zahraei Salehi T, Mahdavi A, Shahroozian E, Salimi Bejestani MR, Mehdizade Mood S, et al. 2018. Distribution of antibiotic resistance genes among the phylogroups of Escherichia coli in diarrheic calves and chickens affected by Colibacillosis in Tehran, Iran. Arch Razi Inst 73: 131137. doi: 10.22092/ari.2018.116502

41. Stockwell VO, Duffy B. 2012. Use of antibiotics in plant agriculture. Rev Sci Tech OIE 31: 199-210. doi: 10.20506/ rst.31.1.2104

42. Stromberg ZR, Johnson JR, Fairbrother JM, Kilbourne J, Van Goor A, Curtiss R, Mellata M. 2017. Evaluation of Escherichia coli isolates from healthy chickens to determine their potential risk to poultry and human health. PLoS One 12: e0180599. doi: 10.1371/ journal.pone.0180599

43. Subedi M. 2018. Antibiotic resistance pattern and virulence genes content in avian pathogenic Escherichia coli (APEC) from broiler chickens in Chitwan, Nepal. BMC Vet Res 14: 113. doi: 10.1186/s12917-018-1442-z

44. Tafur JD, Torres JA, Villegas MV. 2008. Mecanismos de resistencia a los antibióticos en bacterias Gram negativas. Infectio 12: 217-226. 
45. Vetting MW, Hegde SS, Wang M, Jacoby GA, Hooper DC, Blanchard $J S$. 2011. Structure of QnrB1, a plasmidmediated fluoroquinolone resistance factor. J Biol Chem 286: 25265-25273. doi: 10.1074/jbc.M111.226936

46. Zhu Ge X, Jiang J, Pan Z, Hu L, Wang $S$, Wang H, Leung FC, Dai J, Fan H.
2014. Comparative genomic analysis shows that avian pathogenic Escherichia coli isolate IMT5155 (O2:K1:H5; ST complex 95, ST140) shares close relationship with ST95 APEC O1:K1 and human ExPEC O18:K1 strains. PLos One 9: e112048. doi: 10.1371/journal.pone.0112048 